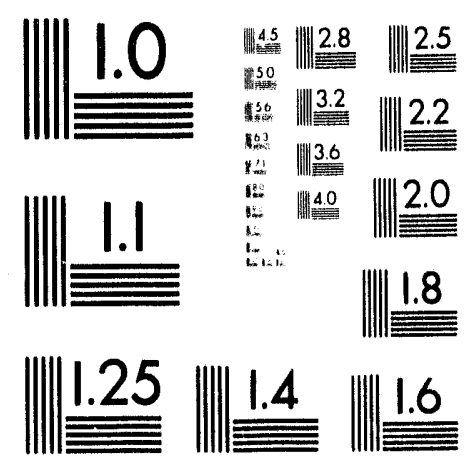



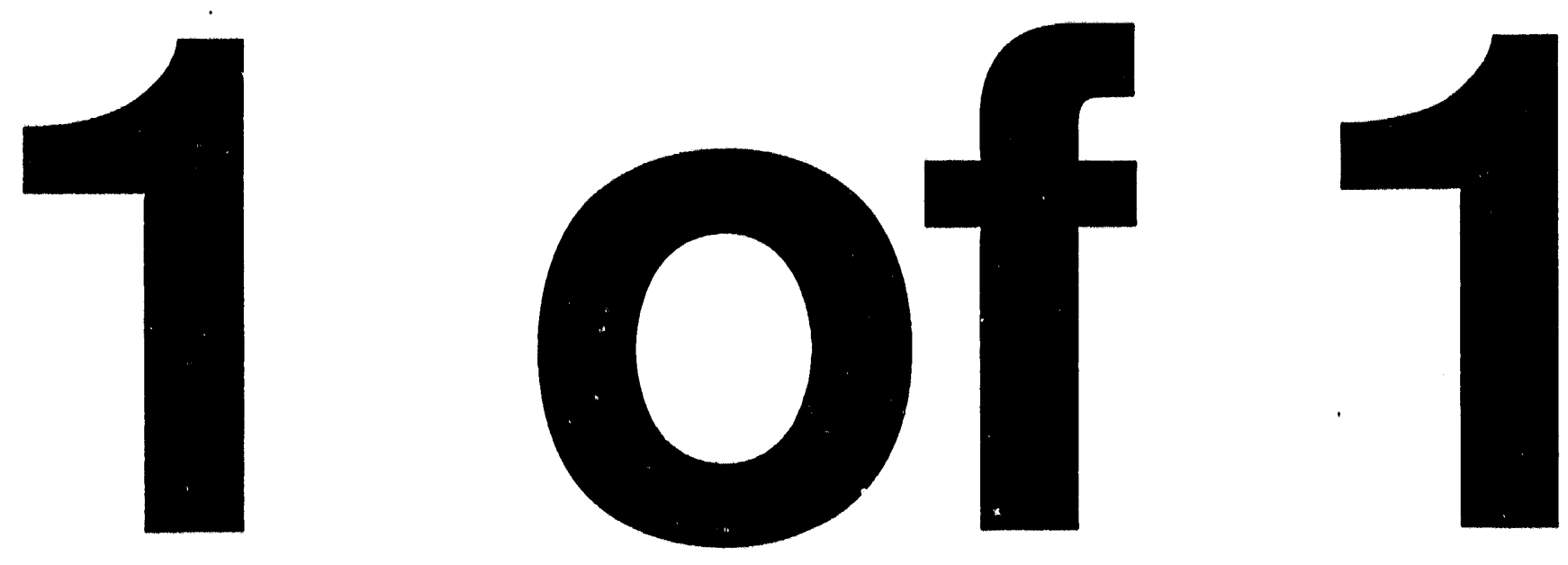


\title{
First Principles Calculations for Analysis Martensitic Transformations
}

\author{
B. N. Harmon, G. L. Zhao, K. M. Ho, C. T. Chan, Y. Y. Ye, Y. Ding, and B. L. Zhang
}

Ames Laboratory* and Department of Physics and Astronomy, Iowa State University, Ames, IA 50011, USA

The change in crystal energy is calculated for atomic displacements corresponding to phonons, elastic shears, and lattice transformations. Anomalies in the phonon dispersion curves of $\mathrm{NiAl}$ and $\mathrm{NiTi}$ are analyzed and recent calculations for TiPd alloys are presented.

\section{INTRODUCTION}

In this paper we briefly cover some of our recent theoretical work which seeks to understand or explain the atomistic driving forces relevant for martensitic transformations. The computations rely on first principles electronic structure methods which have enough precision to yield the changes in total energy as atoms are displaced from one structural phase to another. A few comments about the present and future (parallel computing) methods are given in the next section. Section 3 gives an example of how total energies can aid in understanding transformations at the microscopic level. The roles of the Fermi surface and details of the electronic structure are emphasized using NiTi and TiPd alloys as examples in section 4 .

\section{METHODS}

Band structure calculations employing the local density approximation for exchange and correlation have proven remarkably accurate for evaluating many crystal properties such as lattice constants, bulk modulus, and even phonon frequencies. A recent review has been given by Jones and Gunnarsson [1]. For crystals, one makes use of periodicity, so that for bcc or fcc lattices only one atom per primitive unit cell needs to be considered. For describing martensitic transformations involving the atoms of a crystal shifting cooperatively between one crystal symmetry and another, periodicity can usually be maintained at the expense of including more atoms per unit cell. Since calculations which require the diagonalization of a Hamiltonian matrix increase in difficulty as the number of atoms per cell to the third power (the N3 problem), highly precise calculations are presently limited to systems with 100 or fewer atoms per cell. This is not enough to address the effects caused by some defects (e.g., dislocations or grain boundaries), but it is sufficient to evaluate the important energies for most transformations. Even the N3 problem may present only a temporary road block as new (order $\mathrm{N}$ ) methods are now being developed and will have optimal efficiency with new parallel computers able to handle 1000 's of atoms.

\section{TOTAL ENERGDES, PHONONS, AND PHASE TRANSFORMATIONS}

Martensitic phase transformations involve the cooperative rather than diffusive movement of atoms. For small displacements about their equilibrium positions, such movements of atoms can be analyzed in terms of the normal modes of vibration, or phonons. If the lattice is stiff and resists displacements in certain directions, then the corresponding

\footnotetext{
- This work was performed at the Ames Laboratory which is operated for the U.S. Department of Energy by lowa State University under contract No. W-7405-Eng-82.
} 
phonon frequency is large; however, when the lattice is "ripe" for a phase transformation involving the large displacement of atoms, the phonon with the corresponding displacements usually has a small frequency. Since such transformations commonly occur as the temperature is lowered, the relevent phonons in the high temperature crystal structure often exhibit an anomalous decrease of frequency as the temperature is lowered toward the transformation temperature. Whenever large displacements are involved, and whenever the energy of a single phonon is small compared to the thermal energy $(\mathrm{kT})$, anharmonic effects become important. A key insight arising from detailed theoretical calculations was the importance of the anharmonic coupling of special phonons to the elastic strains which have always been known to play an important role in martensitic transformations. An informative example of this is the bec to hep transformation which occurs in $\mathrm{Zr}$ as a function of temperature, and in $\mathrm{Ba}$ as a function of pressure. A review of these transformations and some of the theoretical calculations has been given previously [2], so here we present only the bare essentials.

To achieve the transformation from the bec to hep phase only two types of displacements or order parameters are required. The first order parameter is the magnitude of the displacement of atoms along directions corresponding to the zone boundary $(\pi / a, \pi / a, 0)$ phonon mode with $(1,-1,0)$ polarization. These displacements correspond
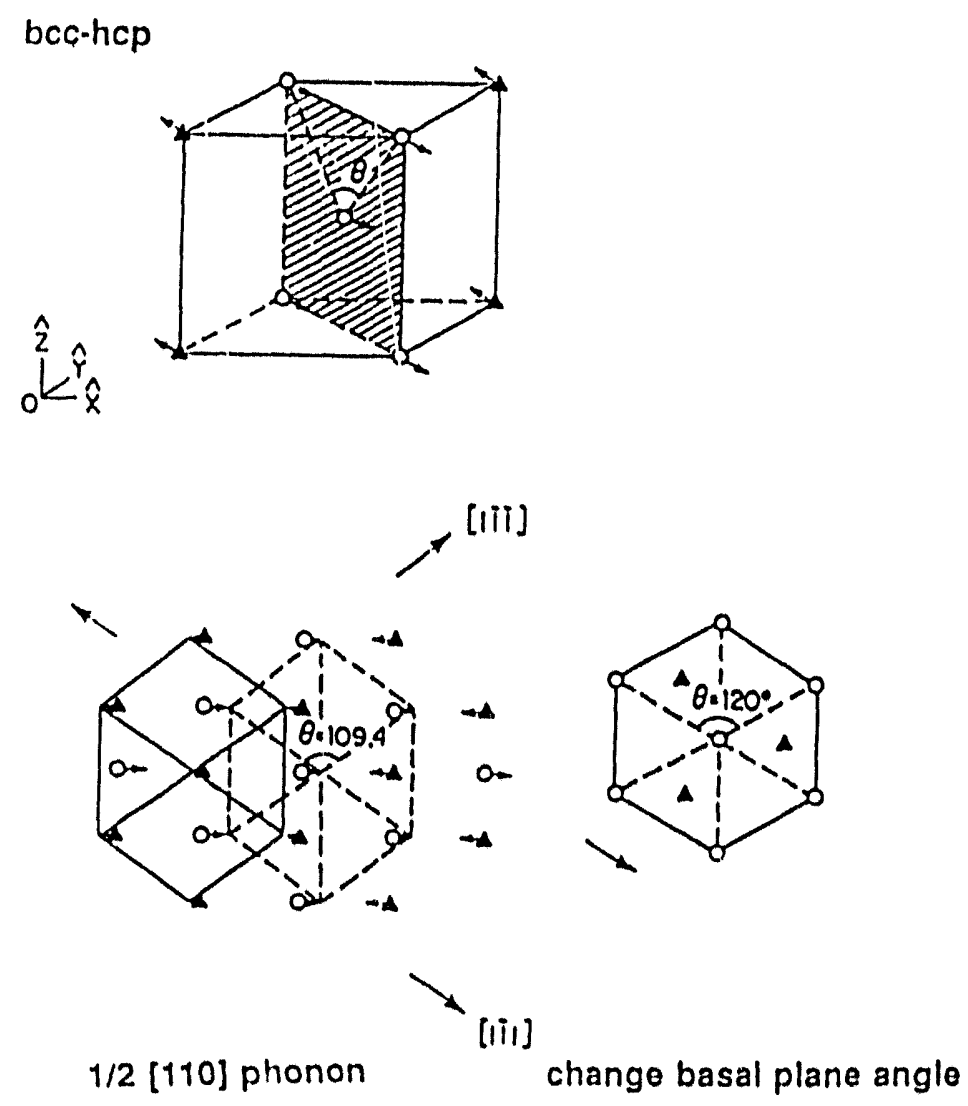

Figure 1. The bcc to hcp phase transition coordinates. The arrows indicate the atomic displacements corresponding to the polarization vector of the relevant $\mathrm{N}$-point phonon in the bcc phase. An uniform shear then changes the angle from $109.47^{\circ}$ to $120^{\circ}$ to obtain the hcp phase. 


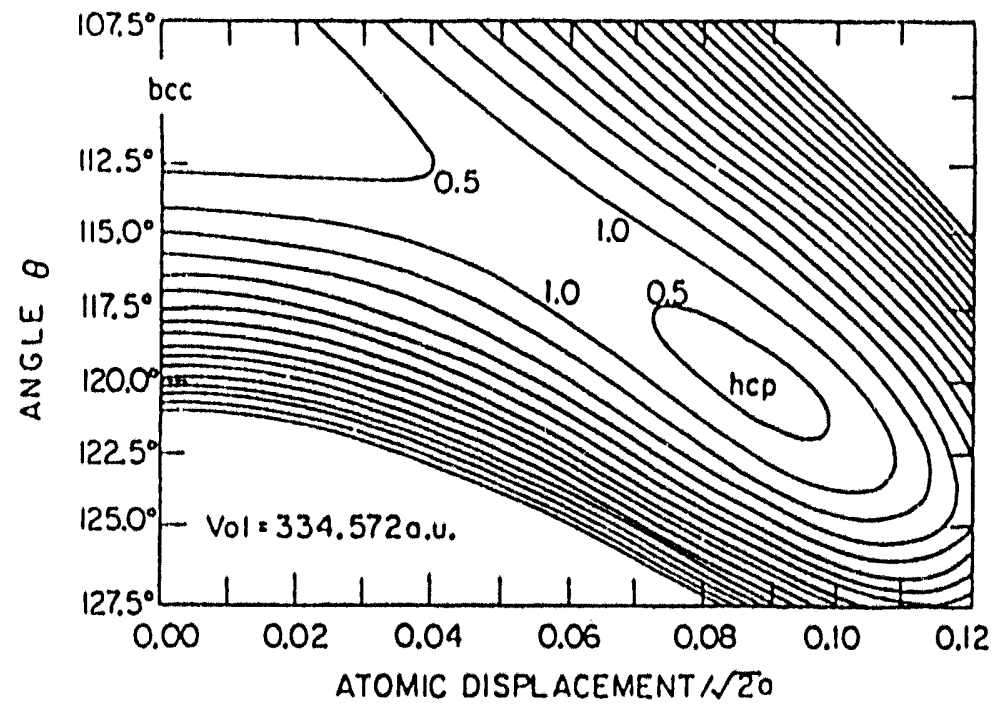

Figure 2. A volume where the bcc and hep energies are nearly equal.

to every other $(1,1,0)$ plane sliding across its neighboring planes as indicated by the arrows in Figure 1. The second order parameter corresponds to a uniform shear or Bain strain that changes the angle $\theta$ shown in Figure 1. The hcp structure is then represented by a displacement of $\delta=(\sqrt{ } 2 / 12) \mathrm{a}$ and an angle of $\theta=120^{\circ}$ as shown. By calculating the total energy at 13 different displacements for the phonon, and 9 different angles, an accurate energy surface was obtained [3]. Figure 2 shows the energy surface for $\mathrm{Ba}$ with contours at $0.5 \mathrm{mRy} / \mathrm{cell}$ intervals $\left(\sim 40^{\circ} \mathrm{K} /\right.$ atom). A low energy barrier between the bce and the hep is evident. A small linear, horizontal displacement from the bcc minimum raises the energy quaratically, from which the phonon frequency may be deduced (in good agreement with experiment). A small vertical displacement from the bcc minimum also raises the energy quadratically and corresponds to the elastic energy. Metallurgists have traditionally focused on the elastic properties when studying such phase transformations while physicists have emphasized phonons. Clearly either of these order parameters by themselves are misleading, and it is their anharmenic coupling at larger displacements which leads to the lowest energy path between the two phases.

As pressure is added the hep minimum is lowered relative to the bcc, and at $\sim 50 \mathrm{kbar}$ the bcc phase is no longer even metastable and the pressure induced transformation will take place even at very low temperatures. At the time the above calculations were performed for $\mathrm{Ba}$, the volume and c/a ratio were not included as order parameters since they differed very little between the two phases. These order parameters are also frequently ignored in Landau expansions of the free energy since they do not include any extra symmetry elements required to go from one lattice structure to the other. The necessity of including such non-symmetry breaking order parameters was made clear by calculations for the bcc to $h c p$ and $9 R$ transformations in $\mathrm{Na}$ [4]. The net effect of these additional order parameters is to lower the energy barrier between phases, making the transformation easier (lowering the transformation temperature or pressure).

We have avoided mentioning temperature as a variable up to now. The energy surface presented was calculated assuming $\mathrm{T}=0^{\circ} \mathrm{K}$ 
where the computations may be performed with high precision. Temperature of course brings in the need to consider a system's free energy and in particular, entropy. By knowing the electronic structure and the vibrational spectra for each of two separate phases of material, the free energies can be evaluated and the stable structure determined as a function of temperature; however, it is not so easy to establish an entropy or an activation barrier when the lattice is half way along the minimum energy path between two phases. At low temperatures it is possible to use $3 \mathrm{rd}$ and 4 th order perturbation theory, and to evaluate the phonon-phonon interaction strengths using first principles total energy calculations [5], however, the effort is large, and the extrapolation to higher temperatures and/or large displacements is problematic. We now believe that molecular dynamics simulations probably offer the best approach for incorporating temperature into the study of free energies and lattice dynamics. This is discussed in the last section.

\section{PHONONS AND SOFT MODES}

As we mentioned in the prev ous section, a low phonon frequency indicates a lack of resistance to particular displacements. It is not surprising, therefore, that most martensitic transformations involve atomic displacements that can be associated with a specific low frequency phonon in the high temperature phase. In some bcc metals and alloys the whole [110] phonon branch is low in energy, and it is not always obvious from observing the measured spectra, which phonon wave vector will be the one associated with the transformation. In many cases, however, the measured phonon spectra do show pronounced phonon anomalies at just those wave vectors that are important for the phase transformation. In this section we consider well known intermetallic alloy systems where this is the case.

$\mathrm{NiAl}$ alloys present an interesting class of
$\mathrm{CsCl}$ structure systems where phonon anomalies have been measured, with a dip occurring in the [110] transverse branch in the vicinity of the wave vector associated with the $7 \mathrm{R}$ periodicity of the martensitic phase [6]. We have made extensive first principles calculations which are able to precisely reproduce the phonon anomaly and have traced its origin to what is called "nesting" on the Fermi surface [7]. The basic idea is that when two flat, parallel pieces of the Fermi surface "nest" (i.e., are separated from each other by a wave vector $\vec{Q}$ ) then the electronic response to phonons with that wave vector is unusually large, an in ideal circumstances the resulting electronic screening of the ionic forces can be strong enough to drive the phonon completely soft (i.e., to zero frequency). In the case of $\mathrm{NiAl}$, the maximum phonon anomaly occurs for $\mathrm{Ni}$ concentrations near $63 \%$, and the resulting compositional disorder smears the Fermi surface enough that the "nesting" is partially destroyed, preventing the phonon from globally going soft. The strength and position of the anomaly depends on composition (in agreement with experiment). If local compositional fluctuations may then result in local soft modes is an open question; one that is of very great interest for understanding the nucleation mechanism.

In the course of the work on NiAl, we theoretically evaluated the phonons for the 50.50 alloy and found two other rather small anomalies along the [100] and [111] directions [7]. These anomalies have just recently been confirmed by highly precise neutron scattering experiments [8], giving more confidence in the theoretical methods and their predictive power.

NiTi alloys are well known for exhibiting the shape-memory effect. In the high temperature $\beta$ phase the entire [110] transverse phonon branch is low in energy, similar to the case of NiAl and many other $\beta$. phase systems. As temperature is lowered the first transformation is to the intermediate or " $R$ " phase, which results from a transverse 
phonon $Q_{0} \simeq(1 / 3,1 / 3,0) 2 \pi / a$ going soft [9]. In an earlier study we showed how Fermi surface nesting quantitatively accounted for this particular phonon anomaly [10]. To demonstrate how dramatic and effective the nesting mechanism can be, we show in Fig. 3 this to be true, but the appearance of the incommensurate phase and its rapid change from $1 / 3$ to $1 / 4$ ordering with Cr contents of $6 \%$ to $10 \%$ was puzzling. Substituting $\mathrm{Cr}$ for Pd acts to add conduction electrons (the stronply correlated d-electrons in Pd act as

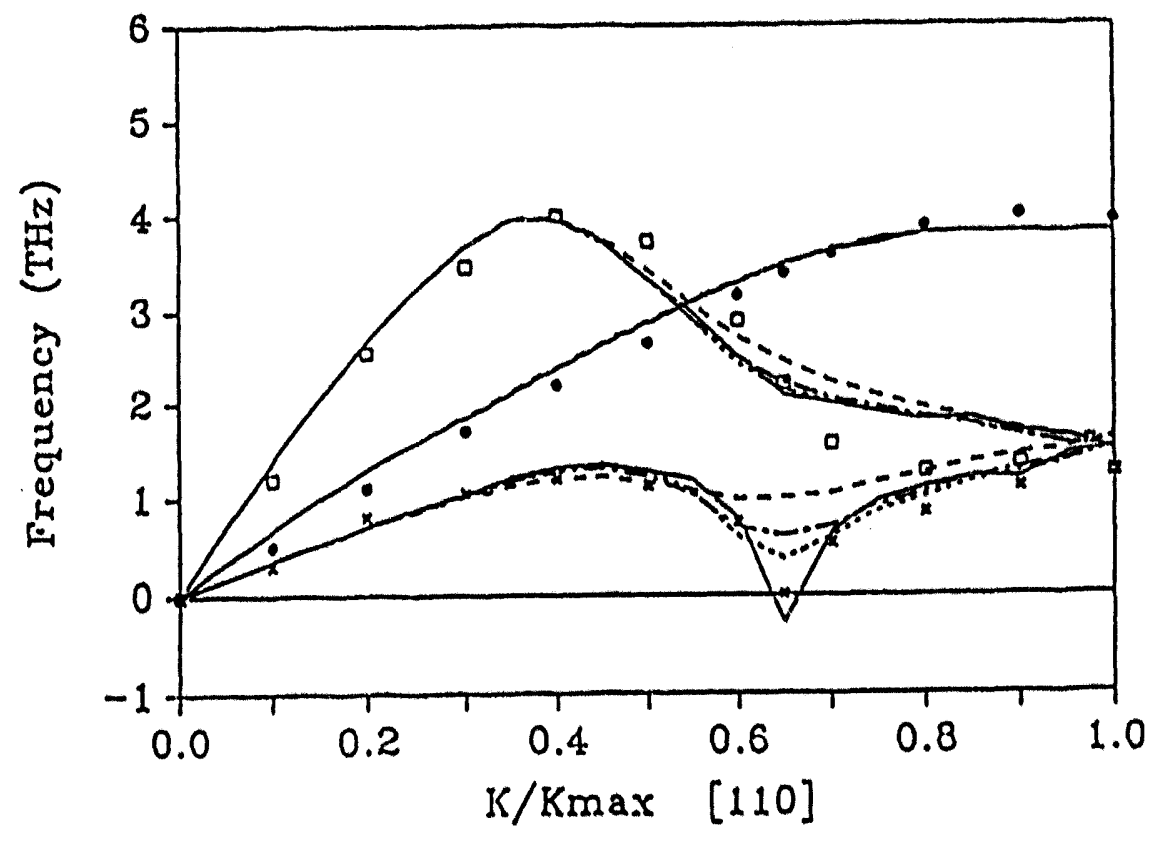

Figure 3. The calculated phonon dispersion curves along the [110] direction for the B2 phase of NiTi. All the symbols are experimental data points. The calculated results are for $\mathrm{T}=0$ $\mathrm{K} ; \ldots . . . ., \mathrm{T}=320 \mathrm{~K} ; \ldots . . ., \mathrm{T}=500 \mathrm{~K}$, and $\ldots \ldots . ., \mathrm{T}=1000 \mathrm{~K}$

the calculated phonon dispersion curves for four different temperatures. As the temperature is raised the Fermi surface is smeared and the phonon anomaly is greatly reduced, just as in the experiment [11]. The theory is described in reference [12].

Just recently we have turned to TiPd alloys and wish to report our preliminary results. When low concentrations of $\mathrm{Cr}$ are substituted for Pd in these alloys a modulated periodicity is observed in a phase similar to the " $R$ " phase of NiTi $[13,14]$. Indeed, since these alloys are isoelectronic one expects the Fermi surfaces to be similar [15]. We find those in $\mathrm{Ni}$, i.e., as core states with about 9 fixed olectrons [7]); however, increasing the number of valence electrons increases the magnitude of the ordering wave vector for the pieces of Fermi surface that are important for the " $R$ " phase in NiTi. Our calculations indicated that for TiPd, there is a new nesting of the Fermi surface which becomes active at about $6 \% \mathrm{Cr}$ concentrations. Above $6 \% \mathrm{Cr}$ the ordering wave vector decreases rapidly (see Fig. 4). These preliminary calculations did not include the electron-phonon matrix elements, which are necessary to assess the magnitude of the phonon anomaly; such 
calculations are more involved and are now being performed to confirm if Fermi surface nesting is indeed the driving force for the incommensurate phase in these alloys.

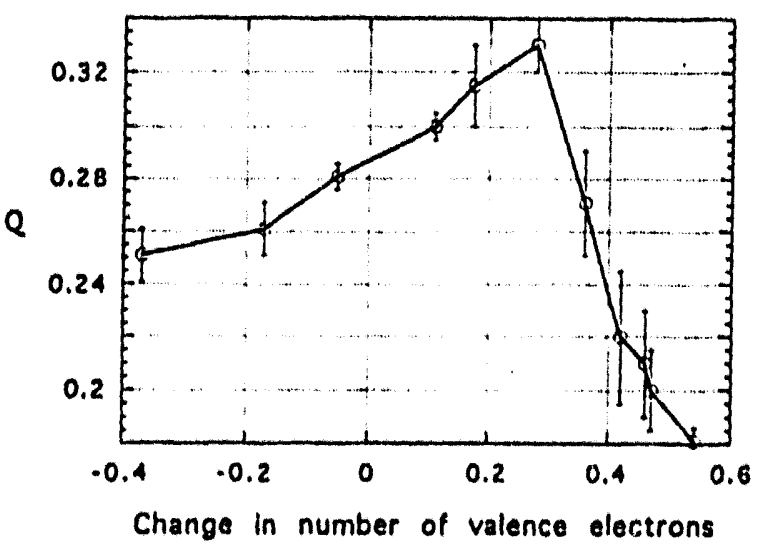

Figure 4. The calculated ordering wave vector $\vec{Q}=(Q, Q, 0) 2 \pi / a$ for the TiPd alloy system as a function of the change in the number of conduction electrons. The peak occurs near the $6 \% \mathrm{Cr}$ alloy.

\section{CONCLUSIONS}

Because of space limitations we have had to limit the above examples. Our own group and several other groups have utilized first principles calculations to study many other martensitic phase transformations. An important point is that although modern calculational techniques are proving accurate and are opening up the quantitative analysis of microscopic interactions, the future is even more promising. The advent of powerful parallel computers with thousands of processors has changed the landscape. Modeling systems with hundreds of thousands of atoms using classical interatomic potentials is becoming standard, and modeling systems with thousands of atoms using the full quantum mechanical interactions is now double. Applications to the nucleation of martensitic transformations, and the role of defects can be expected within a year or two. These are exciting times, and one certainly anticipates new insights, new predictions, and new applications.

The authors would like to thank Lee Tanner for sending them information about TiPd alloys and also for his interest in our work. They also wish to thank Steve Shapiro for sending graphs of his neutron scattering data [8].

\section{REFHRENCES}

1. R. O. Jones and O. Gunnarsson, Rev. Mod. Phys. 61, 689 (1989).

2. K. M. Ho and B. N. Harmon, Mat. Sci. and Eng. A 127, 155 (1990).

3. Y. Chen, K. M. Ho, and B. N. Harmon, Phys. Rev. B 37, 283 (1988).

4. R. J. Gooding, Y. Y. Ye, C. T. Chan, K. M. Ho, and B. N. Harmon, Phys. Rev. B 43, 13626 (1991).

5. Y. Y. Ye, Y. Chen, K. M. Ho, B. N. Harmon, and P. A. Lindgard, Phys. Rev. Lett. 58, 1769 (1987).

6. S. M. Shapiro, B. X. Yang, Y. Noda, L. E. Tanner, and D. Schryvers, Phys. Rev. B 44, 9301 (1991).

7. G. L. Zhao and B. N. Harmon, Phys. Rev. $B$ 45, 2818 (1992).

8. H. Chou and S. M. Shapiro (private communication).

9. See for example S. M. Shapiro, Y. Noda, Y. Fujii, and Y. Yamada, Phys. Rev. B 30 , 4314 (1984) and references therein.

10. G. L. Zhao, T. C. Leung, B. N. Harmon, M. Kiel, M. Müllner, and W. Weber, Phys. Rev. B 40, 7999 (1989).

11. H. Tietze, H. Müllner, and B. Renker, J. Phys. C: Solid State Phys. 17, L529 (1984).

12. G. L. Zhao and B. N. Harmon, Phys, Rev. $B 48$ (in press).

13. K. Enami, K Horil, and J. Takahashi, ISIJ International 29, 430 (1989).

14. A. J. Schwartz and L. E. Tanner, these proceedings.

15. I. I. Naumov, O. I. Velikokhatmyi, and V. Z. Bashirov, JETP Lett. 84, 673 (1991). 


\section{DISCLAMMIXR}

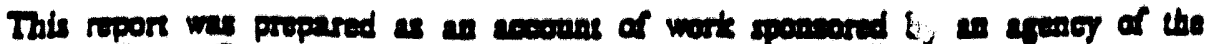

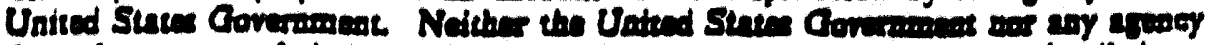

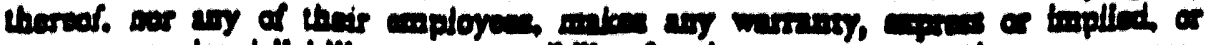

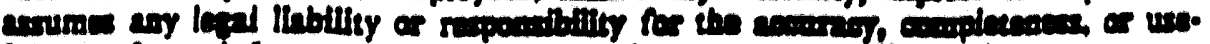

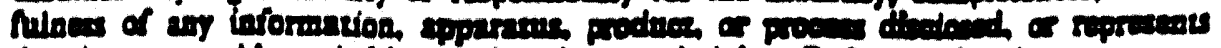

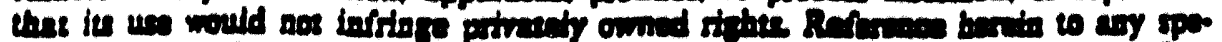

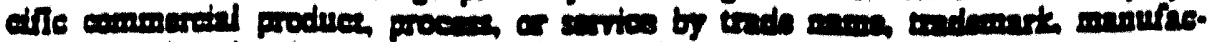

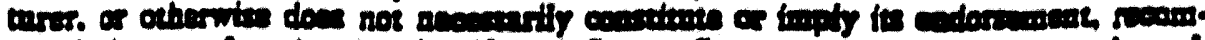

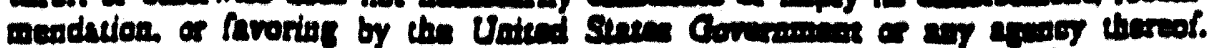

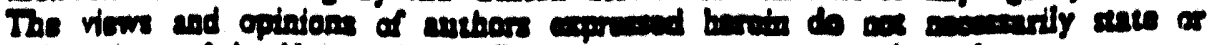

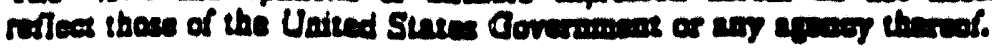



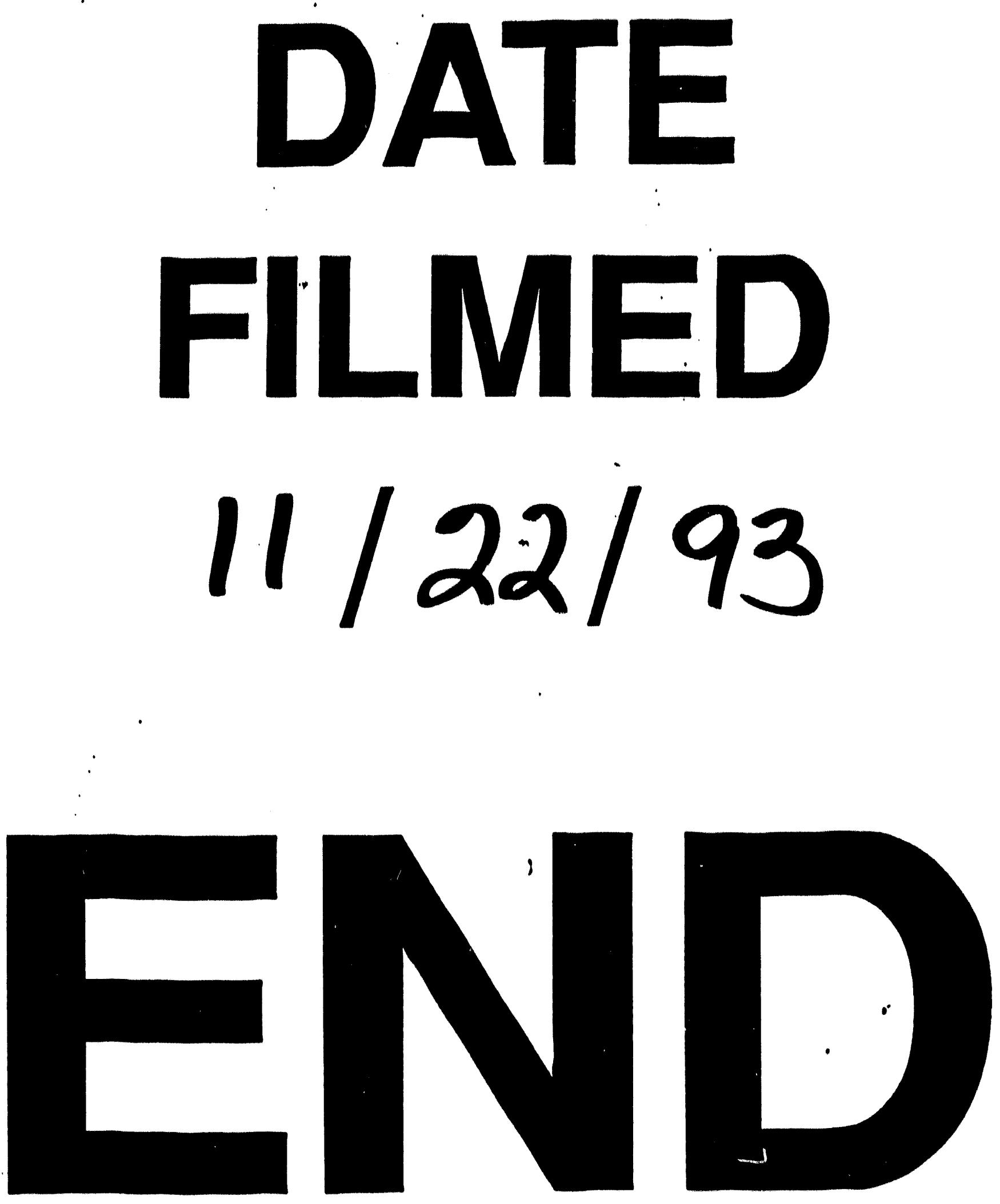


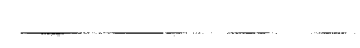

